\title{
Improving efficiency management of radiopharmaceutical materials at a nuclear medicine department
}

\author{
Ali Al Ahmed, Khaled Al-Surimi \\ King Abdulaziz Medical City- Riyadh (KAMC) and King Saud bin Abdulaziz University for Health Sciences, Saudi Arabia
}

\begin{abstract}
The cost of radiopharmaceutical materials is highly expensive compared with other resources employed in nuclear medicine department. Hence, inefficient utilization of these costly materials will lead to waste and more financial burden on the healthcare system, increasing the patient waiting list for important diagnostic procedures, especially in those with need urgent care on time. The available data for the previous 12 months about positron emission tomography / computed tomography (PET/CT) unit at nuclear medicine departments showed that over $16 \%$ of radiopharmaceutical materials were not utilized and being wasted due to increased number of cancelled or rescheduled oncology patients.
\end{abstract}

The overall financial cost for the underutilized radiopharmaceutical materials due to cancelled and rescheduled procedures for 142 patient were about 39,760 US dollar. Most of these are the oncology patients with diabetes arriving at the nuclear medicine department with high blood glucose level and so are not fit for the procedure. This project aims to improve the oncology diabetic patients preparation for PET/CT procedure to avoid wasting the radiopharmaceutical materials. After implementing the PDSA cycles on 14 oncology patients we found that the quantity of not utilized radiopharmaceuticals were significantly reduced. On the other hand, majority of oncology diabetic patients became more aware about the importance of following the required preparation instruction.

\section{Problem}

Reviewing the radiopharmaceutical records at PET/CT unit, nuclear medicine department in King Abdulaziz Medical City (KAMC), Riyadh showed that there was a noticeable percentage $(16 \%)$ of radiopharmaceutical materials being wasted and not utilized due to frequent cancellation of PET/CT procedure on a daily basis. This frequent cancellation had led to an unnecessary waste of very costly materials such as radiopharmaceuticals and resulted in increasing patients' waiting list for the required procedures as the result of the cancellation, and negatively impacted satisfaction level among these vulnerable oncology patients due to delays in their diagnostic and therapeutic plan. This percentage of radiopharmaceutical that was not utilized was audited and a quality improvement project was organised to reduce the percentage of radiopharmaceutical wastes in $\mathrm{PET} / \mathrm{CT}$ unit of nuclear medicine department at KAMC.

\section{Background}

The cost of radiopharmaceuticals in the PET/CT unit is highly expensive compared with other resources employed in nuclear medicine department. In fact, it has a short half life (110 minutes) and any delay in utilizing these materials at specific time will increase the wastes and add more financial burden on the nuclear medicine department. It is worth mentioning that these materials are supplied to our unit from an external supplier in a daily basis, and its quantity was calculated based on the number of scheduled oncology patients on the following day. Therefore, once a patient has cancelled, it is difficult to substitute another oncology patient who is on the waiting list for the same week.

Thus, the high rate of PET/CT daily procedure cancellation will not only increase the financial wastage, but will also cause a systemic disruption to the multidisciplinary level of healthcare services provided to the oncology patients at our hospital. Moreover, it affects the quality of the services provided in nuclear medicine department in general and adds a higher operational cost on the hospital.

Improving the efficiency of healthcare resources is one of the core quality improvement dimensions where this problem can fit in. The $\mathrm{WHO}$, among others, are calling for improvements to accessibility to medical products in a cost-efficient way that avoids wasting resources.[1] Likewise, the main strategies of the Ministry of Health in Saudi Arabia for the next ten years (2010-2020) [2] calls for applying the principles of economics methods and optimal use of medical resources. This means that it was an imperative for us to maximize the utilization of department's allocated resources through practical strategies that will improve the patient preparation in order to make patients ready for the the PET/CT procedure and to avoid rejection of the patient and wasting the radiopharmaceuticals.

\section{Baseline measurement}

A retrospective analysis of all PET/CT procedures at nuclear medicine department for the previous 12 months was performed. Data were collected from both radiopharmaceutical records and picture archiving and communication system (PACS) software, which provides access to all radiological images and a report on 
each cancelled cases. All cancellation and the reasons for them were recorded. One hundred forty two out of $870 \mathrm{PET} / \mathrm{CT}$ cases $(16 \%)$ were cancelled on the same day of procedure. Of these, 56 (39.4\%) were cancelled due to high blood glucose level (>180 $\mathrm{mg} / \mathrm{dL}$ ) measured on the day of procedure. All oncology patients were scheduled earlier through PET/CT unit front disk. The total financial loss for all cancelled procedures was US $\$ 39,760$.

These baseline results shows that significant financial loss from increased percentage of radiopharmaceutical wastes at PET/CT unit. Therefore, we decided to conduct a full cycle of quality improvement aiming for efficient utilization of the allocated resources via reducing the percentage of radiopharmaceuticals wastes with the potential to avoid the higher cancellation rate.

See supplementary file: ds5661.docx - "Main reasons of PET/CT procedure cancellation "

\section{Design}

The Pareto chart showed that (39.4\%) of procedure cancellations resulted from oncology patients with diabetes arriving at PET/CT unit with high blood glucose ( $>180 \mathrm{mg} / \mathrm{dL})$. To gain a better overview of this problem, the quality team decided to review the actual flow chart in the PET/CT unit, starting with receiving PET/CT referral form.

The improvement team at PET/CT unit decided to implement the following change ideas as a result of these findings and reviews: firstly, since oncology patients with diabetes often present a challenge, we need to give simple verbal instructions to these patients before their appointment. This simple instruction was shared with them verbally at the time of booking along with the printed information attached to the appointment slip. The second change idea was testing the process of reviewing and screening $\mathrm{PET} / \mathrm{CT}$ request forms at the time as booking by radiologist to ensure that clinical indications and all required information are clearly stated and matching with procedure requirements.

The improvement measures for these changes were: outcome measure (percentage of reduction in not utilized radiopharmaceuticals in the unit). The process measures includes number of rescheduled patients; number of instructed patients and number of ordered radiopharmaceuticals. Finally, the balancing measure were: patients and physicians satisfaction. The run chart was used to analyze impact of these changes ideas before and after implementation of PDSA cycles.

\section{Strategy}

Before starting the quality improvement cycles, the change ideas were highlighted to the relevant staff in the PET/CT unit including: radiologists, nuclear medicine technologists, nurses, and unit assistance.

PDSA cycle 1: In this cycle we tested the idea of educating oncology diabetic patients by explaining the instruction verbally at the time of booking by one of the staff member in the unit. This step was in addition to the printed information attached to their appointment slip. Our aim at this stage was to have the oncology patients arriving at the unit with blood glucose level $(<180 \mathrm{mg} / \mathrm{dL})$. These instruction includes following a restricted diet, fasting hours, and taking diabetes medication as prescribed on the day before their appointment.

PDSA cycle 2: the second cycle aimed to test the process of reviewing and screening PET/CT referral forms at the time of booking by radiologists to ensure that clinical indications and all required information are clearly stated and matching with procedure requirements. This change idea was considered in order to avoid any possibility of cancellation due to improper booking time, especially for those who had therapeutics sessions recently (chemotherapy or radiotherapy) which is a contraindication for the procedure.

Fourteen PDSA cycles were conducted to test these change ideas. We found a significant reduction in the quantity of radiopharmaceuticals that were not utilized as a result of adherence to the standardized protocols that was shared with diabetic oncology patients. In addition, reviewing the PET/CT request form by radiologists enabled us to manage the specific booking date which matches with patients' treatment plan.

\section{Results}

Baseline measures were collected from both radiopharamcy records and picture archiving and communication system (PACS) software, which provides access to all radiological images and reports on each cancelled cases. The percentage of utilized radiopharmaceuticals was measured following introduction of educating oncology diabetic patients and screening PET/CT request forms at the time of booking. These interventions had resulted in reducing the percentage of radiopharmaceuticals wastes significantly. Although the change ideas were tested for short period, but it shows a positive impact on reduction of radiopharmaceutical wastes and improved our day to day operation.

Based on our data, two to four cancellation events were recorded in daily basis, with the majority coming from oncology diabetic patients. The daily financial loss from these cancellation was between US\$ 560 to US\$1120. The selected improvement change ideas resulted in reducing the percentage of cancellation to $0.4 \%$ which was recorded over the intervention period. Our assumption at this stage that, if we implement these change ideas for a longer period, the cancellation frequency rate in PET/CT unit will be reduced, and we would estimate a significant improvement in radiopharmaceutical utilization.

See supplementary file: ds5659.docx - "Run chart for quantity of utilized Radiopharmaceuticals at PET/CT unit”

\section{Lessons and limitations}

This project has taught us the importance of data and record 
BMJ Quality Improvement Reports

keeping of our daily operation at the unit. Collecting, analyzing, and then interpreting the radiopharmacy data allows us to identify the areas where system is falling short. This allows us to make improvement plans and track the outcomes. In addition, we learnt that recruiting a quality improvement team with diversity in terms of skills, experience, and knowledge is essential to have different viewpoints that led to an efficient work. Furthermore, close monitoring of our daily operation at PET/CT unit including radiopharmaceuticals ordering and data recording by one of staff member is necessary to ensure sustainability. The positive outcomes of this project taught us that these simple interventions can be reflected on other radiopharmacy activities in nuclear medicine department aiming for better resource management.

There were some challenges faced the QI team during the course of this project, and it was suspected at this stage of testing different change ideas. Inability to reach the referring physician due to missing contact number on the PET/CT referral form caused some delay in conforming the detailed clinical indications and screening information. Moreover, some of the oncology diabetic patients were not available at the time of booking and the team had to share the required preparation and procedure instruction with their family member. These two issues resulted a delays in issuing and confirming the PET/CT appointment and caused service dissatisfaction among view oncology patients. However, the staff at $\mathrm{PET} / \mathrm{CT}$ unit were supportive and worked together to achieve the aim of this project and considered every step as a learning opportunity that may unveil areas for future improvement.

\section{Conclusion}

The high cost of radiopharmaceuticals is a significant part of the annual budget of PET/CT unit and nuclear medicine department in general. In fact, it is a valuable target for quality improvement projects that aims to reduce the percentage of wastes and improves resource management at work site. Applying the model for improvement from finding the performance gap, then choosing the right measures and testing the change ideas on different PDSA cycles was a powerful strategy that helped us in achieving our aim in this project.

However, this achievements needs a close monitoring for all day to day operations at PET/CT unit to ensure its sustainability. One of the suggested ideas to achieve this goal is to have a close monitoring of radiopharmacy daily activities by a senior staff member to ensure the efficient utilization of all requested radiopharmaceuticals.

\section{References}

1. World Health Organization (2007). Everybody's business: Strengthening health systems to improve health outcomes: WHO's framework for action. Geneva: World Health Organization.

2. About the Ministry - Strategy. (n.d.). Available from: http://www.moh.gov.sa/en/Ministry/About/Pages/Strategy.as px

\section{Declaration of interests}

There are no conflicts of interest.

\section{Acknowledgements}

Edna Samtang

PET/CT unit Charge Nurse

\section{Ethical approval}

There was no need for ethical approval since this project aims primary to bring about improvement not to generate new knowledge for research purpose. The Nuclear Medicine department management supported conducted this quality improvement project for the benefits of improving both the resources management and quality of patients care. 